\title{
Intimal Angiosarcoma of the Aorta: Single Case Report and Effective Surgical Management
}

\author{
Merveesh Luveanand Auchoybura ${ }^{\mathrm{a}}$, Cunhua Su${ }^{\mathrm{a}}$, Xin Chen ${ }^{\mathrm{a}}$, \\ Fuhua Huang ${ }^{\mathrm{a}, \mathrm{b}}$
}

\begin{abstract}
Primary malignant tumors of the aorta (PMTAs) are not only extremely rare, but also exhibit enormous histological heterogeneity. We present the first recorded case of a female patient with intimal angiosarcoma of the aortic arch and descending thoracic aorta in China, along with the clinical presentation, histopathologic diagnosis, and surgical excision of the lesion. We propose surgery as a viable curative approach to angiosarcomas of intimal origin.
\end{abstract}

Keywords: Aorta; Angiosarcoma; Surgical management

\section{Introduction}

Primary malignant tumors of the aorta (PMTAs) are a group of extremely rare tumors affecting the aorta with around 200 cases reported cases up until now. The biological behavior of these tumors is highly aggressive, with a median survival time of only a few months. Distal and peripheral thromboembolic complications are the typical clinical presentation. Several authors recommend resection of the tumor-bearing aortic region $[1,2]$, but there is no proof that this approach is of any benefit to the patient. Chemotherapy and radiotherapy have proven to be of less value for the patient's survival, but may play a role in certain circumstances where the patient is inoperable or metastatic complications have ensured [3]. We surgically treated a female patient diagnosed with angiosarcoma who was admitted to our center in June 2015. The patient was healthy with no complications or new malignant lesions after 1-year follow-up of her case.

Manuscript submitted September 21, 2017, accepted October 11, 2017

aDepartment of Cardiovascular Surgery, Nanjing Hospital Affiliated to Nanjing Medical University, Nanjing Cardiovascular Disease Research Institute, Nanjing, Jiangsu 210006, China

${ }^{b}$ Corresponding Author: Huang Fuhua, Department of Cardiovascular Surgery, Nanjing Hospital Affiliated to Nanjing Medical University, Nanjing Cardiovascular Disease Research Institute, Nanjing, Jiangsu 210006, China. Email: mauchoybur@yahoo.com

doi: https://doi.org/10.14740/jcs332w

\section{Case Report}

A female patient was admitted to our hospital with dyspnea on June 4, 2015. She reported that she had been feeling increasingly short of breath for the past 6 months along with bilateral weakness of the lower extremities for the last 4 months. Her past medical history was inconsistent for claudication. She had a 6-year history of hypertension, for which she was not undergoing treatment. The patient underwent radical hysterectomy for endometrial cancer in the year 2005 and radical colonectomy for colon cancer in the year 2007. In both instances, she had not been treated with adjuvant chemotherapy or radiotherapy. No recurrence or metastasis had been detected on clinical follow-up. On examination, there was a significant difference in blood pressure of the right upper limb (177/88 mm Hg) and lower limb (132/67 mm Hg). The pulse in the pedalis dorsum arteries were palpable bilaterally. Pre-operative computed tomography angiogram (CTA) of the thorax and abdomen was inconclusive, indicating possible chronic aortic dissection (Fig. 1). Brain CT scan was negative for embolic and metastatic lesions. No abnormalities in liver function tests, biochemistry, urea and electrolytes, kidney function tests, and other laboratory investigations were discovered.

\section{Surgical method}

After induction of general anesthesia, a right subclavian incision was made and the right axillary artery was exposed followed by an inguinal incision to expose the femoral artery and median sternotomy. The right axillary artery and femoral artery were both cannulated and a dual stage atrio-caval cannula was placed in the right atrium. Right axillary artery was used for cardiopulmonary bypass (CPB) and selected cerebral perfusion (SCP). The arterial line was bifurcated (line 1 and line 2). Line 1 was used for axillary artery perfusion. Line 2 was again bifurcated (line 3 and line 4). Line 3 was used for perfusing branch cannulation and line 4 was used for femoral artery perfusion. The patient was cooled to a naso-pharyngeal temperature of $22-24{ }^{\circ} \mathrm{C}$ and cold blood cardioplegia and ice were routinely used for myocardial protection. During cooling, the brachiocephalic trunk, left common carotid artery and left subclavian artery were dis- 


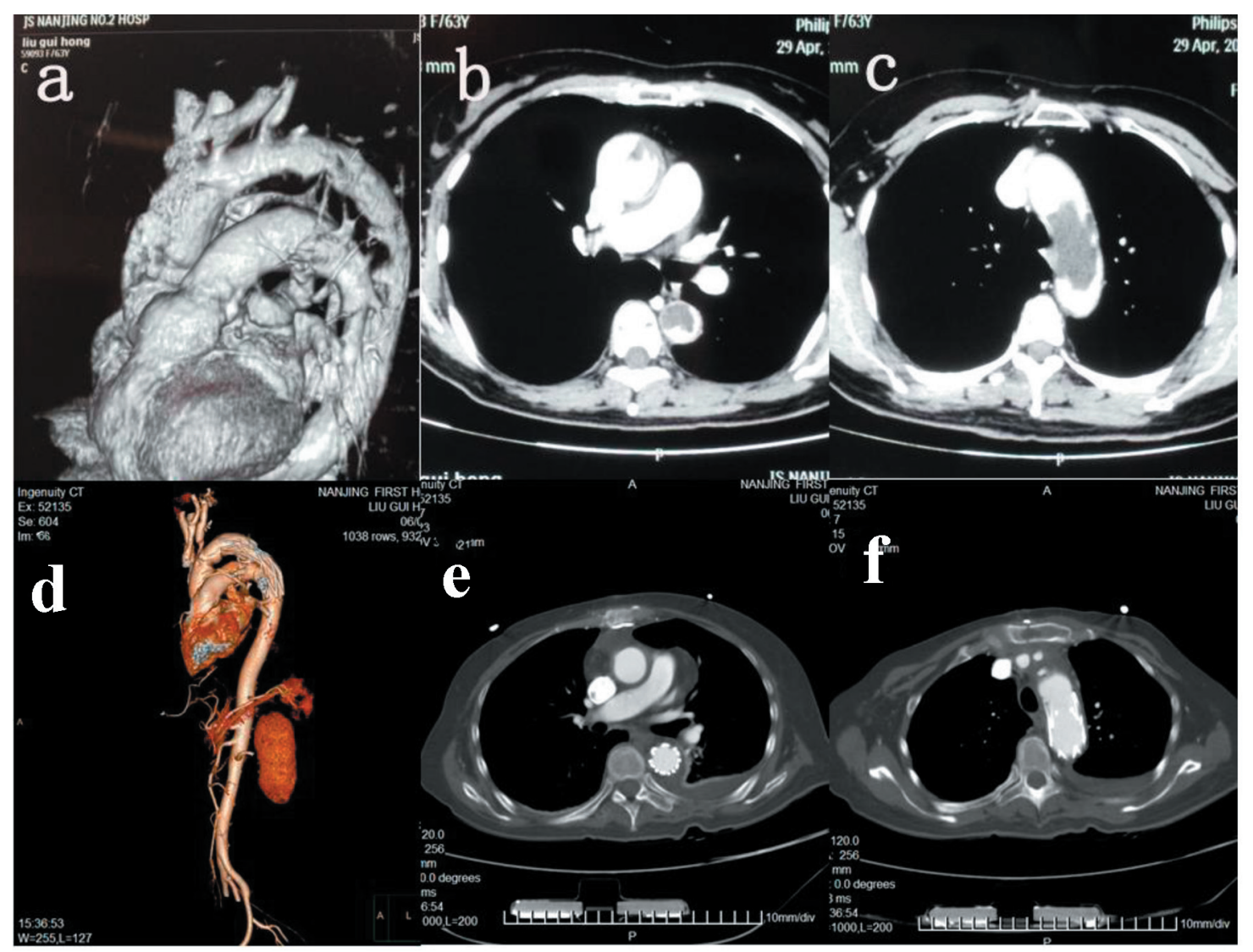

Figure 1. CT and HRCT. (a) CTA 3D imaging of aorta pre-operatively. (b) HRCT descending aorta pre-operatively. (c) HRCT aortic arch pre-operatively. (d) CTA 3D imaging of aorta post-operatively. (e) HRCT descending aorta post-operatively. (f) HRCT aortic arch post-operatively.

sociated. The ascending aorta was clamped at the distal end and the proximal segment was transected up to the sinotubular junction. After careful examination of root structures, we decided to use the Bentall procedure for reconstruction. The branches of the aortic arch were clamped and CPB was discontinued when the nasopharyngeal temperature dropped to $22{ }^{\circ} \mathrm{C}$, while the brain continued to be perfused at a rate of approximately $5 \mathrm{~mL} / \mathrm{kg} / \mathrm{min}$ through the right axillary artery. The transverse aortic arch was transected between the left carotid artery and left subclavian artery. The part of the aortic wall containing the lesion was also simultaneously excised. The posterior mediastinum was accessed through an incision in the posterior wall of the pericardium. A small incision was made in the wall of the descending thoracic aorta. Using a rubber band, the descending thoracic aorta distal to the incision was tied. The whole segment of the thoracic aorta from the transected arch to the tied off end was flushed with water, after which we untied the descending aorta, and closed both incisions. A catheter sheath contaning the stented graft was inserted into the appropriate segment of the descending aorta, and the stented graft was deployed. The distal end of the tetrafurcation graft was anatomosed to the proximal end of the stent, and antegrade systemic perfusion was immediately established through the perfusion branch. The left common carotid artery was anatomosed to the graft side branch to establish dual brain perfusion and rewarming was started. The proximal end of the branched graft was anatomosed to the distal end of the ascending aorta and the ascending aorta was unclamped. The left subclavian artery and brachiocephalic trunk were successively anastomosed to the graft side branches. An ascending aorta, right femoral artery bypass concluded the procedure.

\section{Discussion}

\section{Introduction}

PMTAs are not only extremely rare, but also aggressive mesenchymal neoplasms. Based on their origin and clinical characteristics, Wright et al have suggested categorizing these tumors as either intimal or mural [4]. The intimal type often forms intraluminal polyps or extends along the lumen, causing peripheral emboli or aortic obstruction. The mural type originates from the media or adventitia and usually extends extramurally to para-aortic tissues and lymph nodes [5-7]. Although the incidence of angiosarcomas is extremely low, they are nevertheless the most common type of primary cardiac malignancy and are usually noted in men between the ages 


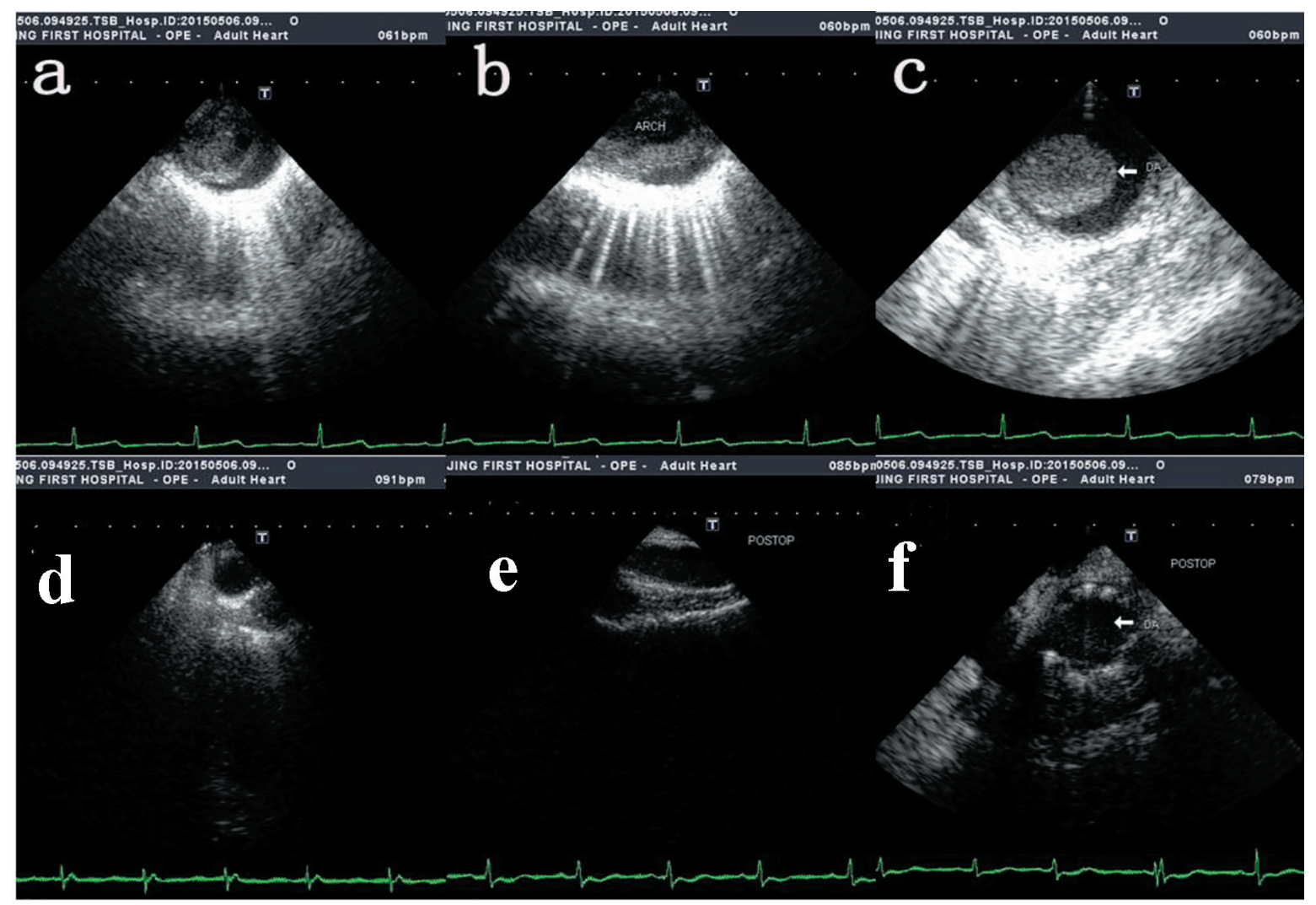

Figure 2. Intra-operative transesophageal echocardiography showing tumor mass protruding into the lumen. (a, b, c) The preoperative transesophageal echocardiography of ascending aorta, aortic arch and thoracic descending aorta respectively. (d, e, f) The post-operative transesophageal echocardiography of ascending aorta, aortic arch and thoracic descending aorta respectively.

of 20 and 50 with about $75 \%$ arising from the right atrium or pericardium. Brodowski reported the first case of PMTA and since then, less than 200 cases have been reported. The male/ female ratio is 9:5 [8] and cases have been reported across all ages, in newborns (at 3.5 months) as well as in the elderly (85 years old). No cases of intimal angiosarcoma of the aorta have been reported in China to date. In a pooled analysis performed by Rusthoven et al, undifferentiated tumor histology was the most common $(65 ; 39.4 \%)$, followed by vascular or angiomatous $(22 ; 13.3 \%)$, and fibro/myoblastic $(12 ; 7.3 \%)$. The most common pathological site of origin was the aortic intima (110; $66.7 \%)$, followed by unspecified site $(41 ; 24.8 \%)$, and mural origin $(14 ; 8.5 \%)$ [9].

\section{Molecular background}

From a molecular point of view, intimal angiosarcomas have yet to be analyzed at the genetic level. The development of sarcomas in relation with the abnormal expression of the p53 gene [10], as well as angiosarcomas arising after implantation of dacron grafts has been documented [11]. However, the specific mechanism of disease is unclear. Thalheimer et al postulated that the MDM-2/p53 pathway is instrumental in the pathogenesis of intimal sarcoma, whereby $20 \%$ of tumor cells were positive for $\mathrm{p} 53$, implicating this pathway. BodeLesniewska et al also report overexpression of the p53 gene in intimal angiosarcomas. Moreover, in the study published by Stewart et al, the Akt/mTOR/pathway, STAT3 pathway, COX-2 and CD-44 were also reported to be implicated in the biology and development of high-grade sarcoma of the aorta [12].

\section{Characteristic clinical features on presentation}

Due to the rarity of intimal sarcomas of the aorta and late manifestation of the disease, clinical diagnosis is often challenging. The symptoms associated with PMTAs are non-specific and elusive, sometimes mimicking atherosclerotic disease of the aorta and peripheral arterial disease, and at other times, renal colic, or herniated nucleus pulposus. Aortic angiosarcomas usually present with clinical signs related to embolization of tumor fragments or metastasis of the primary tumor [9]. The most frequent presentations are acute aortic embolization (20.6\%), claudication (18.8\%), abdominal discomfort (12.7\%) and back pain $(9.1 \%)$, and about $44.8 \%$ of patients have metastatic disease on presentation. CT scans and MRI are both used for imaging and although some authors prefer MRI for its ability to detect arterial wall sarcomas [13], transesophageal 


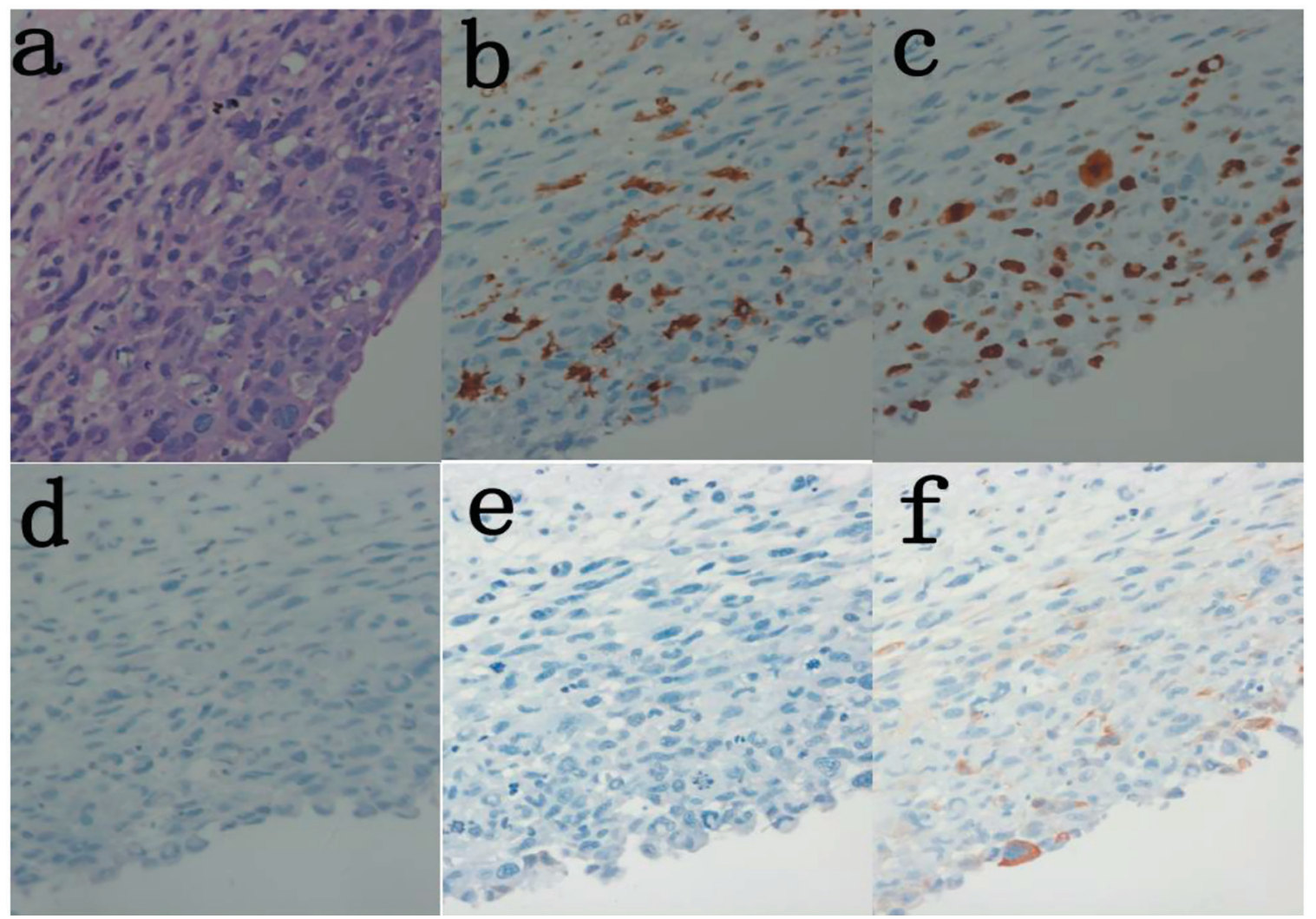

Figure 3. Histolopathology of excised aortic wall lesion shows malignant infiltration of smooth muscle cells. (a) H\&E $(\times 400)$. (b) Immunoassay Ki-67 (30\%) (× 400). (c) Immunoassay CD68(+) (× 400). (d) Immunoassay CD31(-) (× 400). (e) Immunoassay $\operatorname{SMA}(-)(\times 400) .(f)$ Immunoassay caldesmon (-) (× 400).

echocardiography remains the modality of choice for early diagnosis (Fig. 2) [14]. Histologic examination of the specimen is mandatory as it confirms the diagnosis (Fig. 3). Intraoperative frozen section analysis assures adequate resection within clear margins [10].

\section{Therapeutic management}

When dealing with intimal angiosarcomas, comprehensive approches should be employed to assure the best possible outcome. Surgery, with resection of the lesion and replacement with artificial grafts is an effective method to slow down the progression of the disease, or in some cases completely arrest its progression $[15,16]$. With surgery, the life expectancy is $25.5 \%$ at 40 months [9]. Surgery combined with adjuvant chemotherapy and radiotherapy has proven to be beneficial in that it increases the life expectancy of the patient $[17,18]$. Additionally, tumor cells have the ability to activate the coagulation system [19], and thromboembolic events are rather common as a clinical presentation or complication following surgery. Treatment to prevent thromboemboli can also be considered in selected patients.

The prognosis for primary aortic tumors is poor with an average survival rate of 15.6 months after diagnosis; the mean survival rate for intimal angiosarcomas is 9.8 months [20]. On follow-up after 12 months, the patient treated at our center was still alive and free of complications, with no evidence of recurrence or metastasis.

\section{Conclusion}

To our knowledge, this is the first case report of intimal angiosarcoma of the aorta in China. Although extremely rare, aortic angiosarcomas are fatal with low life expectancy at 1 year. Surgical resection is a viable option for treatment of intimal angiosarcomas of the aorta.

\section{Conflict of Interest}

The authors declare that there is no conflict of interest regarding the publication of this paper.

\section{Grants}

Jiangsu Provincial Special Program of Medical Science (BE2017610). Department of Thoracic and Cardiovascular Surgery, Nanjing First Hospital, Nanjing Medical University, 68, Nanjing 210006, Jiangsu, China. 


\section{References}

1. Seelig MH, Klingler PJ, Oldenburg WA, Blackshear JL. Angiosarcoma of the aorta: report of a case and review of the literature. J Vasc Surg. 1998;28(4):732-737.

2. Weiss WM, Riles TS, Gouge TH, Mizrachi HH. Angiosarcoma at the site of a Dacron vascular prosthesis: a case report and literature review. J Vasc Surg. 1991;14(1):8791.

3. Burke AP, Virmani R. Sarcomas of the great vessels. A clinicopathologic study. Cancer. 1993;71(5):1761-1773.

4. Wright EP, Glick AD, Virmani R, Page DL. Aortic intimal sarcoma with embolic metastases. Am J Surg Pathol. 1985;9(12):890-897.

5. Shirani S, Soleymanzadeh-Ardabili M, Arami M. Intimal sarcoma of the descending aorta. Arch Iran Med. 2007;10(2):253-254.

6. Salm R. Primary fibrosarcoma of aorta. Cancer. 1972;29(1):73-83.

7. le Rochais JP, Icard P, Coffin O, Galateau F, Khalil M, Maiza D. Intimal sarcoma of the thoracic aorta: a case report. Eur J Vasc Endovasc Surg. 1999;18(2):181-182.

8. Akiyama K, Nakata K, Negishi N, Henmi A. Intimal sarcoma of the thoracic aorta; clinical-course and autopsy finding. Ann Thorac Cardiovasc Surg. 2005;11(2):135138.

9. Rusthoven CG, Liu AK, Bui MM, Schefter TE, Elias AD, Lu X, Gonzalez RJ. Sarcomas of the aorta: a systematic review and pooled analysis of published reports. Ann Vasc Surg. 2014;28(2):515-525.

10. Thalheimer A, Fein M, Geissinger E, Franke S. Intimal angiosarcoma of the aorta: report of a case and review of the literature. J Vasc Surg. 2004;40(3):548-553.

11. Schmehl J, Scharpf M, Brechtel K, Kalender G, Heller $\mathrm{S}$, Claussen $\mathrm{CD}$, Lescan M. Epithelioid angiosarcoma with metastatic disease after endovascular therapy of ab- dominal aortic aneurysm. Cardiovasc Intervent Radiol. 2012;35(1):190-193.

12. Stewart B, Manglik N, Zhao B, Buryanek J, Khalil K, Aronson JF, Buja LM. Aortic intimal sarcoma: report of two cases with immunohistochemical analysis for pathogenesis. Cardiovasc Pathol. 2013;22(5):351-356.

13. Pompilio G, Tartara P, Varesi C, Biglioli P. Intimal-type primary sarcoma of the thoracic aorta: an unusual case presenting with left arm embolization. Eur J Cardiothorac Surg. 2002;21(3):574-576.

14. Mecklai A, Rosenzweig B, Applebaum R, Axel L, Grossi E, Chan A, Saric M. Intimal sarcoma in the aortic arch partially obstructing the aorta with metastasis to the brain. Tex Heart Inst J. 2014;41(4):433-436.

15. Garg N, Lewis MA, Maleszewski JJ, Kalra M. Intimal sarcoma in an inflammatory aneurysm after endovascular aneurysm repair. J Vasc Surg. 2012;55(4):1134-1137.

16. Hales SL, Locke R, Sandison A, Jenkins M, Hamady M. Aortic angiosarcoma: a rare cause for leaking thoracic aneurysm. Cardiovasc Intervent Radiol. 2011;34(Suppl 2):S20-24.

17. Italiano A, Delva F, Mathoulin-Pelissier S, Le Cesne A, Bonvalot S, Terrier P, Trassard M, et al. Effect of adjuvant chemotherapy on survival in FNCLCC grade 3 soft tissue sarcomas: a multivariate analysis of the French Sarcoma Group Database. Ann Oncol. 2010;21(12):2436-2441.

18. O'Connor JM, Chacon M, Petracci FE, et al. Adjuvant chemotherapy in soft tissue sarcoma (STS): a metaanalysis of published data. J Clin Oncol. 2008;26(Suppl 15): 10526.

19. Caine GJ, Stonelake PS, Lip GY, Kehoe ST. The hypercoagulable state of malignancy: pathogenesis and current debate. Neoplasia. 2002;4(6):465-473.

20. Seelig MH, Oldenburg WA. Regarding "Primary aortic intimal sarcoma of the endothelial cell type with longterm survival”. J Vasc Surg. 1998;28(4):756. 